\section{Long-Term Clinical Follow-Up of Therapeutic Internal Carotid Artery Occlusion}

I read with interest and congratulations the results of "Long-Term 3T MR Angiography Follow-Up after Therapeutic Occlusion of the Internal Carotid Artery to Detect Possible de Novo Aneurysm Formation," 1 as well as the opinion regarding management of internal carotid artery (ICA) aneurysms in this day of new intravascular options.

The authors suggest, with accuracy, that no (or little) systematic follow-up data are available on such patients and that publications are anecdotal. We did systematically review our clinical, if not imaging, follow-up experience with therapeutic occlusion of the ICA, with attention to delayed complications of subarachnoid hemorrhage (SAH) and infarct following balloon occlusion of the ICA. ${ }^{2}$ Sixty of 62 patients (97\%) who survived at least 6 months (mean, 60 months; median, 112 months) were the basis for analysis. We did use MR angiography (MRA) in the follow-up of patients, but not systematically, and with far less than the $3 \mathrm{~T}$ MRA quality available to de Gast et al. ${ }^{1}$ The total follow-up period was 468 patient years. Two SAHs due to de novo anterior communicating aneurysms occurred, and these are mentioned in de Gast's article. The incidence of delayed SAH due to de novo aneurysm was 0.4 per 100 patient-years follow-up. We estimated this occurrence $(400 / 100,000)$ to be 40 times the risk of SAH in the general population and 5 times greater than Miller et $\mathrm{al}^{3}$ suggested might be expected in patients with previous SAH. Perhaps the 109 patient years that de Gast et al reviewed are insufficient to further document this low occurrence rate.

Unfortunately, our manuscript has been lost to the ages in the abyss of nonindexed, short-lived journals, a lesson in itself in scientific documentation methods and an additional caveat to those entertaining thoughts of new journal development.

\section{References}

1. de Gast AN, Sprengers ME, van Rooij WJ, et al. Long-term 3T MR angiography follow-up after therapeutic occlusion of the internal carotid artery to detect possible de novo aneurysm formation. AJNR Am J Neuroradiol 2007;28:508-19

2. Tomsick TA, van Loveren H, Tew JM, et al. Delayed complications of balloon occlusion of the internal carotid artery for unclippable aneurysms. Journal of Neurovascular Disease 1997;2:64-67

3. Miller CA, Hill SA, Hunt WE. "De novo” aneurysms. Surg Neurol 1985;24:173-80

T. Tomsick

Department of Radiology

University of Cincinnati Hospital

Cincinnati, Ohio

DOI 10.3174/ajnr.A0680

\section{Computer-Based 3D MR Imaging Analysis in Amyotrophic Lateral Sclerosis: Common and Specific Factors Among Studies}

Computerized MR imaging analysis including automatic measurements of whole-brain atrophy and the use of unbiased analysis techniques for regional atrophy without prior restriction of the search volume (such as voxel-based morphometry $[\mathrm{VBM}]$ ) is a rapidly growing field in neuroimaging. Recently, Mezzapesa et $\mathrm{al}^{1}$ reported global brain atrophy (ie, reduced brain parenchymal fraction $[\mathrm{BPF}]$ ) in a group of 16 patients with amyotrophic lateral sclerosis (ALS); and in the analysis of local brain changes by VBM in the same patients, they reported gray matter reductions in extramotor areas, including the bilateral frontal and temporal lobes. Both with respect to the global brain atrophy measure and to the regional findings, however, additional comparisons with previous 3D MR imaging studies in ALS are necessary beyond those discussed by Mezzapesa et al.

In a previous study of 22 patients with definite ALS, ${ }^{2}$ BPF assess- ment by use of the same technical approach but a different software solution (statistical parametric mapping [available at www.fil.ion. ucl.ac.uk/spm/] versus Structural Image Evaluation, Using Normalization, of Atrophy [available at www.fmrib.ox.ac.uk/analysis/research/siena] in Mezzapesa et $\mathrm{al}^{1}$ ) came to the same result of a highly significant global brain atrophy compared with 22 age-matched controls. Obviously, whole-brain atrophy as assessed by BPF seems to be an ALS-associated feature, as now demonstrated by 2 independent studies in a total of 38 patients. It is remarkable that this effect could only be observed in the analysis of BPF as the size-normalized proportion of brain parenchymal volume to total intracranial volume, whereas neither the calculation of normalized brain volume in the same study ${ }^{1}$ nor the absolute volume measurements in non-normalized 3D MR imaging data of ALS patients ${ }^{3}$ nor the earlier MR imaging studies cited by Mezzapesa et $\mathrm{al}^{1}$ led to significant group differences between ALS brains and those of controls. Thus, BPF seems to be the most sensitive MR imaging-based biologic marker in this motor neuron disease and has to be further evaluated in a longitudinal design.

With respect to the regional distribution of this atrophy, the pattern and extent of volume losses particularly in frontal and temporal areas vary widely among studies within the well-known overlap of ALS and frontotemporal lobar degeneration (FTLD). Even if detailed neuropsychological investigations as a conditio sine qua non in patient characterization were performed, the results would be heterogeneous. In agreement with Mezzapesa et al, ${ }^{1}$ Grosskreutz et $\mathrm{al}^{4}$ reported extramotor volume losses in parietal and frontal areas in another recent VBM study of 17 patients with ALS, whereas the results of the VBM analysis by Kassubek et $\mathrm{al}^{2}$ of patients with ALS without any neuropsychological/behavioral signs of FTLD with only 1 very small frontal area of gray matter loss were apparently at odds with those of Mezzapesa et $\mathrm{al}^{1}$ and Grosskreutz et $\mathrm{al}^{4}$. Even if methodologic factors might be of relevance, such as the smoothing during data preprocessing (the use of $12-\mathrm{mm} \mathrm{FWHM}^{1}$ versus $6 \mathrm{~mm}^{2}$ increases the sensitivity and reduces the specificity), the exact patient characterization with respect to the clinical presentation and disease-related factors is of utmost importance. For the white matter, the structural brain alterations along the corticospinal tracts were only observed in 1 study so far. ${ }^{2}$

In summary, the morphologic fingerprint of ALS in motor and extramotor brain areas assessable by in vivo 3D MR imaging analysis awaits further investigations in larger homogeneous patient groups; and for the establishment of BPF as a biologic marker at group level, studies in longitudinal design as far as they may be applicable in this rapidly progressive disorder are required.

\section{References}

1. Mezzapesa DM, Ceccarelli A, Dicuonzo F, et al. Whole-brain and regional brain atrophy in amyotrophic lateral sclerosis. AJNR Am J Neuroradiol 2007;28:255-59

2. Kassubek J, Unrath A, Huppertz HJ, et al. Global brain atrophy and corticospinal tract alterations in ALS, as investigated by voxel-based morphometry of 3-D MRI. Amyotroph Lateral Scler Other Motor Neuron Disord 2005;6:213-20

3. Pinkhardt EH, van Elst LT, Ludolph AC, et al. Amygdala size in amyotrophic lateral sclerosis without dementia: an in vivo study using MRI volumetry. BMC Neurol 2006;6:48

4. Grosskreutz J, Kaufmann J, Fradrich J, et al. Widespread sensorimotor and frontal cortical atrophy in amyotrophic lateral sclerosis. BMC Neurol 2006;6:17

$$
\begin{array}{r}
\text { A. Unrath } \\
\text { J. Kassubek } \\
\text { Department of Neurology } \\
\text { University of Ulm } \\
\text { Ulm, Germany }
\end{array}
$$

DOI 10.3174/ajnr.A0681 